\title{
Structural Engineering Studies on Reinforced Concrete Structure using Neutron Diffraction
}

\author{
Hiroshi Suzuki ${ }^{1, a}{ }^{*}$, Koichi Kusunoki ${ }^{2, b}$, Manabu Kanematsu ${ }^{3, c}$, Tomohisa Mukai $^{4, d}$ \\ and Stefanus Harjo, \\ ${ }^{1}$ Materials Sciences Research Center, Japan Atomic Energy Agency, Tokai, Naka, Ibaraki, \\ Japan \\ ${ }^{2}$ Earthquake Research Inst., Univ. of Tokyo, Bunkyo-ku, Tokyo, Japan \\ ${ }^{3}$ Dept. of Architecture, Tokyo Univ. Sci., Noda, Chiba, Japan \\ ${ }^{4}$ Department of Structural Engineering, Building Research Inst., Tsukuba, Ibaraki, Japan \\ ${ }^{5}$ J-PARC Center, Japan Atomic Energy Agency, Tokai, Naka, Ibaraki, Japan \\ a suzuki.hiroshi07@jaea.go.jp, ${ }^{b}$ kusunoki@eri.u-tokyo.ac.jp, ${ }^{c}$ manabu@rs.noda.tus.ac.jp \\ d__mukai@kenken.go.jp, ${ }^{\mathrm{e}}$ stefanus.harjo@j-parc.jp
}

\section{Keywords: Neutron Diffraction, Reinforced Concrete, Transverse Rib, Bond Stress}

\begin{abstract}
It has been demonstrated in our past studies that neutron diffraction can be an alternative method to conventional strain gauges for measuring the stress distribution along rebar embedded in concrete. The current study investigated the possibility of the bond stress evaluation using neutron diffraction in order to find a further capability of neutron diffraction for the structural engineering study on the reinforced concrete structure. Several peaks appeared in the bond stress distribution measured by neutron diffraction, showing the inhomogeneous bond variation along the embedded rebar. This result suggests that the neutron diffraction technique with high spatial resolution makes it possible to investigate local bond resistance caused by the transverse ribs. The bond stress distribution measured by the neutron diffraction technique is expected to bring detailed understanding of the bond mechanism between rebar and concrete for the reinforced concrete structure.
\end{abstract}

\section{Introduction}

The reinforced concrete (RC), which is widely utilized for various architectural and civil engineering structures, is well known as a composite structure, in which concrete with relatively low tensile strength and ductility is strengthened by reinforcements such as steel rods (rebars) with high tensile strength and/or ductility. In general, quantitative evaluation of bond resistance between rebar and surrounding concrete is important to discuss the performance of the RC structures [1-4]. In our previous studies, we have investigated the potential of the neutron diffraction technique for the stress measurement of rebar embedded in concrete as an alternative method to the conventional strain gauge. Our first relevant work was carried out using the engineering diffractometer RESA-1 in JRR3 (Japan Research Reactor No. 3), and we demonstrated that the neutron diffraction technique can be a novel strain measurement method for rebar embedded in concrete [5, 6]. More recently, threedimensional deformation behavior of the embedded rebar including the axial and transverse strains was successfully measured under pull-out loading using Time-of-Flight (TOF) neutron diffraction with the engineering diffractometer, TAKUMI in MLF (Materials and life Science Experimental Facility) of J-PARC (Japan Proton Accelerator Research Complex) [7]. Furthermore, it was demonstrated by some application studies using TAKUMI that the neutron diffraction technique is available to assess the bond deterioration due to rebar corrosion and crack generation in concrete [8]. As described above, our previous studies commonly discussed on the bond condition between rebar 
and concrete based on a change in the axial stress distributions along the embedded rebar measured by neutron diffraction. However, most of the papers investigating the bond strength for the RC structures have rather discussed on a change in the "bond stress distribution" rather than the axial stress distribution $[9,10]$.

The current study was, therefore, undertaken to evaluate the bond condition of the RC structure based on the bond stress distribution along the embedded rebar, in order to find further capability of neutron diffraction for the structural engineering study of the RC structure.

\section{Experimental Procedure}

RC Specimens. Figure 1 (a) shows a schematic illustration of the RC specimen used in this study. A ferritic steel deformed-bar with $9.53 \mathrm{~mm}$ in a nominal diameter, so-called D10 rebar, was embedded in cylindrical concrete with approximately $50 \mathrm{~mm}$ in diameter and $460 \mathrm{~mm}$ in length. The length of the bonded region is $320 \mathrm{~mm}$, and an un-bonded region with $110 \mathrm{~mm}$ in length was artificially introduced at the loading edge of the specimen. This un-bonded region was utilized for determining the reference lattice parameter with stress-free condition. The boundary between bonded and un-bonded regions was defined as the origin of length scale $(X=0)$. The maximum size of the mixed aggregates in the concrete was $13 \mathrm{~mm}$ in diameter. This specimen was cured in air for total 29 days before the neutron experiment, i.e. first 14 days at ambient condition and last 15 days in a constant temperature $\left(20 \pm 1^{\circ} \mathrm{C}\right)$ and humidity $(60 \pm 5 \mathrm{RH} \%)$ room. The compressive and tensile strengths of concrete cured in air for 50 days under the same condition as the reinforced concrete specimen were measured as a reference to be $34.6 \mathrm{MPa}$ and $2.79 \mathrm{MPa}$, respectively. The specimen was mounted on the pull-out loading device which consists of a hydraulic center-hole jack, a center-hole load cell and coil spring, as shown in Fig. 1 (b).

Neutron Experiment. The engineering diffractometer, TAKUMI [11], installed at BL19 in MLF of J-PARC was employed in this study. Figure 1 (c) shows the schematic layout of TAKUMI for the stress measurement of rebar embedded in concrete. This is an energy dispersive neutron engineering diffractometer, which measures energy spectrum diffracted from a sample when irradiating with white pulsed neutrons at 25 $\mathrm{Hz}$ generated in the mercury target. The incident neutron beam was formed into a rectangular shape with $5 \mathrm{~mm}$ in width and 10 $\mathrm{mm}$ in height by using a gauge definition slit, irradiating the reinforced concrete specimen mounted on the loading device settled on a $\mathrm{XYZ} \theta$ positioner. The specimen with the loading device was oriented $45^{\circ}$ to the incident beam, and therefore allowed us to measure the strains of rebar in the axial and transverse directions simultaneously using both detector banks installed at $\pm 90^{\circ}$. The radial collimators for $5 \mathrm{~mm}$ gauge width were installed in front

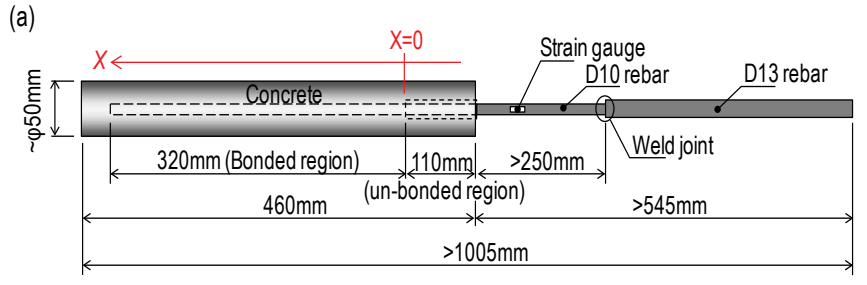

(b)

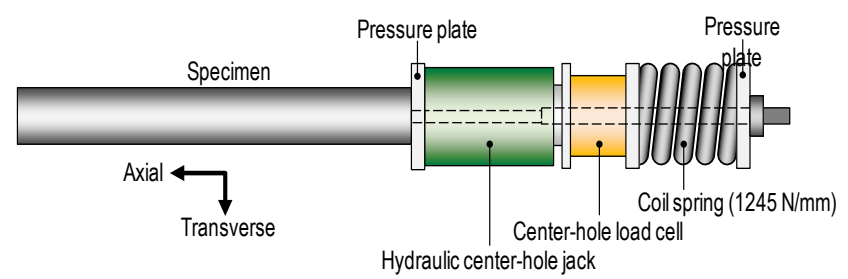

(c)

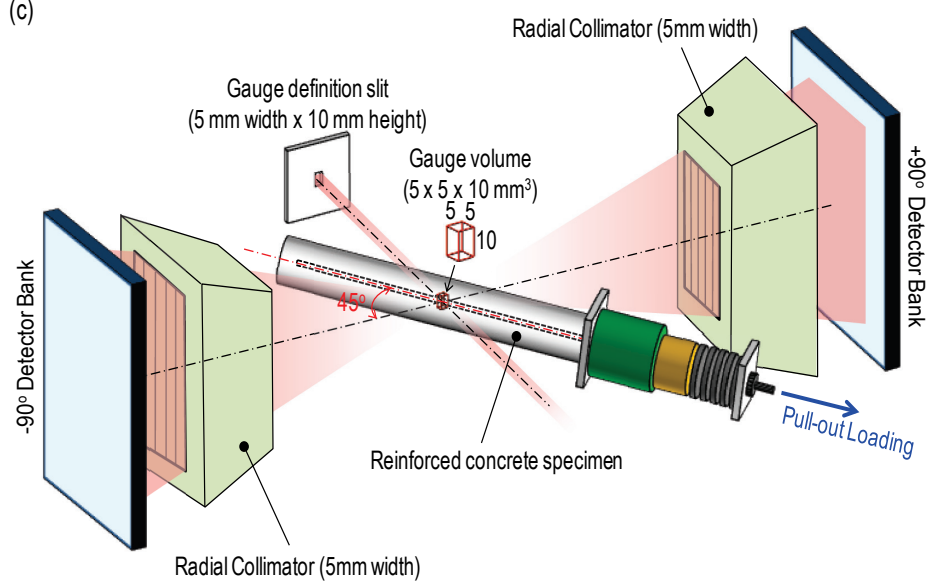

Fig. 1 Schematic illustrations of (a) sample used in this stidy, (b) load frame and (b) measurement configulation of TAKUMI. 
of each detector bank, resulting in the gauge volume of $5 \times 5 \times 10 \mathrm{~mm}^{3}$. The diffraction patterns were measured at $5 \mathrm{~mm}$ intervals in minimum along rebar over the length of 250-300 mm including the first $50 \mathrm{~mm}$ un-bonded region under different pull-out loadings, i.e. approximately $125 \mathrm{MPa}$ and 248.5 $\mathrm{MPa}$. The detailed descriptions of the experimental condition including the sample preparation can be found in the reference [7].

Data Analysis. The average lattice constants, $a$ in the axial and transverse directions were determined by multiple-fitting over 15 peaks from the 110 to 431 reflections by the Rietveld refinement software, Z-Rietveld [12]. The axial stress in a rebar axis, $\sigma_{\mathrm{A}}$ was calculated using the strains in the axial and transverse directions of rebar in assumption of equiaxial-stress condition [7]. On the other hand, the bond stress, $\tau$ is equivalent to the shear stress acting at the boundary between rebar and concrete, expressed by following equation,

$$
\tau=\frac{A}{\pi D} \cdot\left|\frac{d \sigma_{A}}{d X}\right|=\frac{D}{4} \cdot\left|\frac{d \sigma_{A}}{d X}\right|,
$$

where, $A$ and $D$ indicate a nominal cross-sectional area and a nominal diameter of rebar, respectively. According to Eq (1), the bond stress distribution can be obtained by making a differential curve of the axial stress distribution. In this study, the binomial smoothing was, at first, applied to the measured axial stress distribution to reduce the influence of data scattering, and then the bond stress distribution was obtained by plotting slopes fitted within a range of neighboring data in the smoothed axial stress distribution, as a function of the axial position of rebar.

The average bond stress was, in our previous studies [5-8], roughly calculated by Eq (1) with the slope of the axial stress distribution within a range of the anchorage region, approximately estimated by eye. In contrast, it was obtained accurately in this study normalizing the area of the bond stress diagram in the anchorage region by its length.

\section{Results and Discussion}

Figure 2 shows the axial stress distributions and corresponding bond stress distributions along the embedded rebar in concrete under two different pullout loadings, i.e. $\sigma_{\mathrm{ap}}=125 \mathrm{MPa}$ and $248.5 \mathrm{MPa}$. Figure 2(a) exhibits the axial stress distribution with a spatial resolution of $5 \mathrm{~mm}$ in minimum, showing typical stress variation for the RC structure under pull-out loading as an increase in the tensile stress towards the end of the bonded region $(X=0 \mathrm{~mm})$. In addition, compressive residual stresses generated due to drying shrinkage of concrete can be found in the bonded region. The bond stress distribution derived from the smoothed axial stress distribution is shown in Fig. 2(b). Lengths of the anchorage region can be predicted to be about $85 \mathrm{~mm}$ for $\sigma_{\mathrm{ap}}=125 \mathrm{MPa}$ and about $145 \mathrm{~mm}$ for $\sigma_{\mathrm{ap}}=248.5 \mathrm{MPa}$, and the average bond stresses are calculated to be about $6.4 \mathrm{MPa}$ and 5.9 $\mathrm{MPa}$, respectively. These values are different from rough estimation in our previous study [7], but more reliable since the bond stress distribution obtained here assists to determine these values accurately. On the other hand, several peaks can be
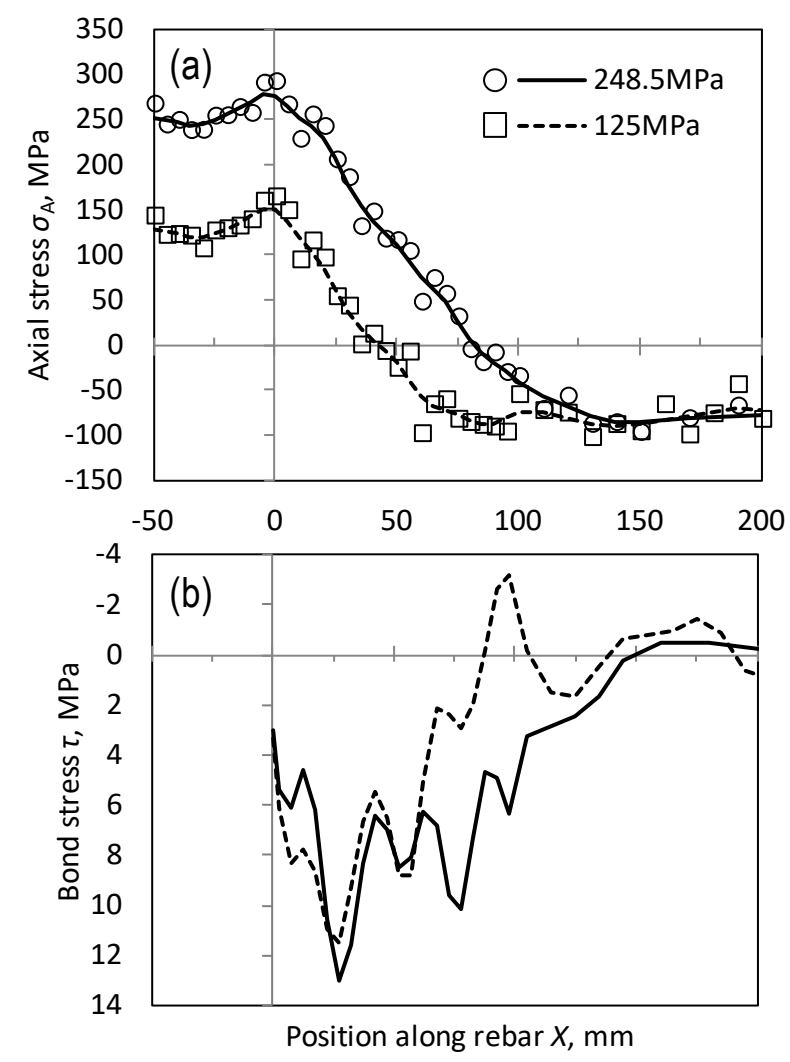

Fig. 2 (a) Axial stress and (b) bond stress distributions with $5 \mathrm{~mm}$ in spatial resolution. Average error bar of the measured axial stresses is $\pm 22 \mathrm{MPa}$. 

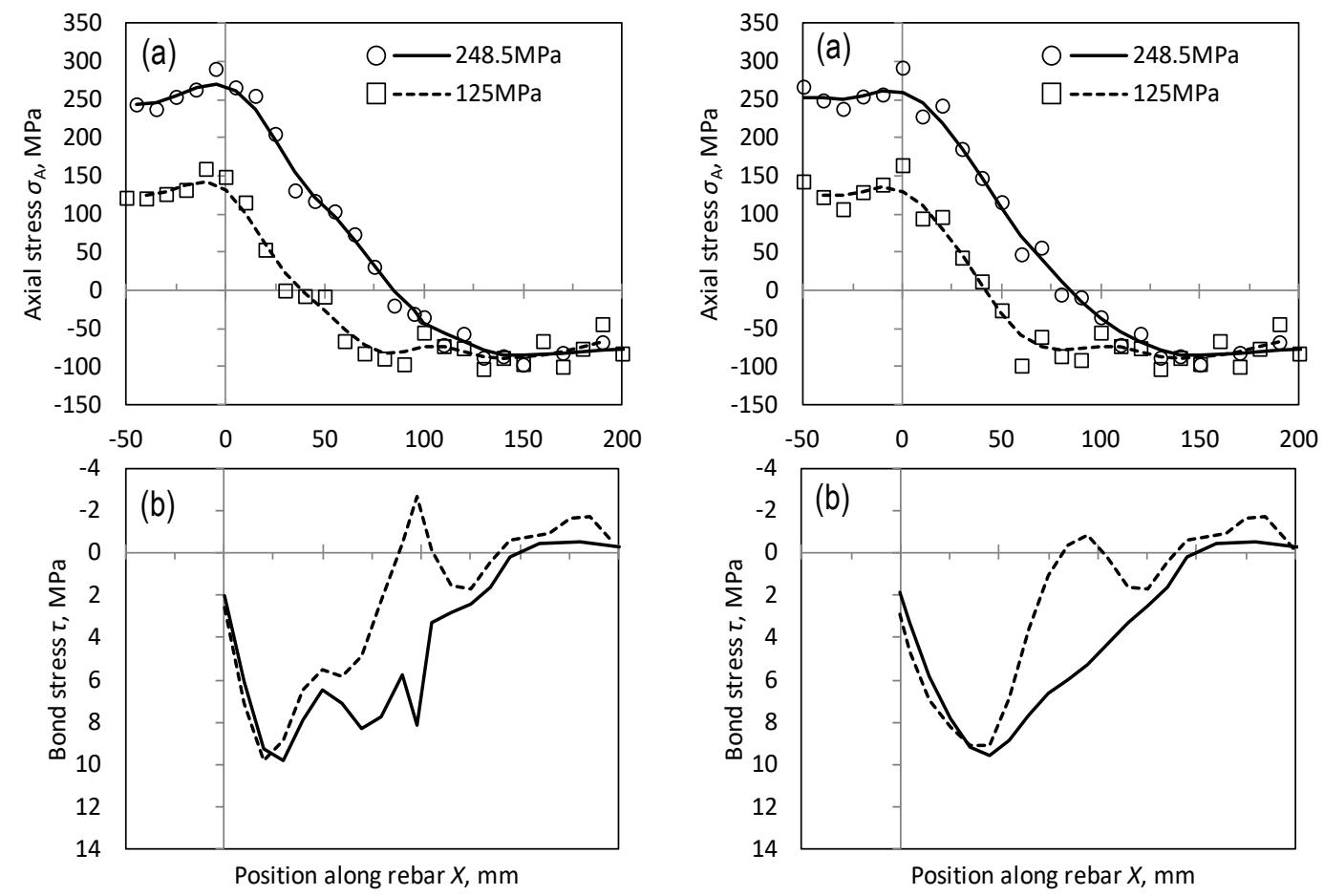

Fig. 3 (a) Axial stress and (b) bond stress distributions with $10 \mathrm{~mm}$ in a spatial resolution.

Fig. 4 (a) Axial stress and (b) bond stress distributions with $10 \mathrm{~mm}$ in spatial resolution, but it is $5 \mathrm{~mm}$ offset from that in

found in the bond stress distribution with about $20 \mathrm{~mm}$ in intervals. Since the error bars of the bond stresses are estimated to be more than $\pm 10 \mathrm{MPa}$, the oscillations in Fig. 2(b) is suspected to be given by the scatter in the axial stresses. However, the cyclic variation showing similar pattern regardless of the applied stresses may be rather associated with ribs regularly distributed along rebar. To know the reason why several peaks appear in the bond stress distribution, therefore, following discussions are made based on the geometrical relationship between the transverse rib and measurement positions.

Figure 3 (a) shows the axial stress distribution with $10 \mathrm{~mm}$ in a spatial resolution, extracted from the original axial stress distribution shown in Fig. 2(a). The bond stress distribution obtained here looks similar pattern to the variation of Fig. 2(b), but showing some broader peaks. The average bond stresses are calculated to be about $5.8 \mathrm{MPa}$ for both applied stresses, which are slightly smaller than those derived from the bond stress distribution with $5 \mathrm{~mm}$ in a spatial resolution. On the other hand, Fig. 4 (a) shows the axial stress distribution with the same spatial resolution of $10 \mathrm{~mm}$, but it is $5 \mathrm{~mm}$ offset from that in Fig. 3(a). As shown in Fig. 4(b), the bond stress distribution derived from the axial stress distribution has no peaks, which is clearly different from the variations shown in Figs. 2(b) and 3(b). The average bond stresses are calculated to be about $5.8 \mathrm{MPa}$ for $\sigma_{\mathrm{ap}}=125 \mathrm{MPa}$ and about 5.6 $\mathrm{MPa}$ for $\sigma_{\mathrm{ap}}=248.5 \mathrm{MPa}$, which are similar value to those in former case with $10 \mathrm{~mm}$ in a special resolution. Comparing the average bond stresses for all cases with different special resolutions, it can be confirmed that they agree within less than $1.0 \mathrm{MPa}$, regardless of the special resolution.

The results showing different trend of the bond stress distribution can be explained by the position relationship between the transverse ribs and measurement positions, as represented in Fig. 5(a). The distance between transverse ribs of D10 rebar is approximately $6.7 \mathrm{~mm}$. If the rib position coincides with the measurement position at $X=10 \mathrm{~mm}$, they are approximately overlapped every $20 \mathrm{~mm}$ when measuring the axial stress distribution with $5 \mathrm{~mm}$ in a spatial resolution, as shown in (i) in Fig. 5(a). Simply assuming a step-like axial stress distribution along the embedded rebar, the axial stress 
distribution measured with $5 \mathrm{~mm}$ in a spatial resolution can be represented by the red line in the bottom figure of Fig. 5(a). In this case, unchanged flat variation can be observed every $20 \mathrm{~mm}$, which brings a decrease in the bond stresses at corresponding positions. This simple model can approximately demonstrate the bond stress distribution shown in Fig 2(b). On the other hand, if the stress distribution is measured with $10 \mathrm{~mm}$ in a spatial resolution starting from $X=5 \mathrm{~mm}$, the measurement positions cannot be overlapped with the rib positions at all (see (ii) in Fig. 5). In this case, the axial stress distribution fluctuates every $10 \mathrm{~mm}$, showing similar trend to the case (i) in Fig. 5(a) but lower resolution, resulting in broader peaks in the bond stress distribution as shown in Fig. 3(b). If starting measurement from $X=0 \mathrm{~mm}$ with $10 \mathrm{~mm}$ in a spatial resolution, on the other hand, the measurement positions can be overlapped with another ribs every $20 \mathrm{~mm}$, as shown in (iii) in Fig. 5. In this case, the axial stress distribution behaves similar trend to the case (ii) in Fig. 5(a), but seems to be shifted by $5 \mathrm{~mm}$. Therefore, the bond stress distribution in Fig. 4(b) shows slightly different from that in Fig. 3(b). Considering above mechanisms, neutron diffraction can investigate local bond resistance taking advantage of higher spatial resolution than the strain gauge method. According to Fig. 5(b), the horizontal bearing force, $F_{\mathrm{H}}$ on the rib face is estimated to be smaller than the friction force, $f_{\mathrm{H}}$ on a straight part of rebar because of higher axial restriction of deformation around the transverse rib by the surrounding concrete. Therefore, lower and higher bond stresses in Fig. 2(b) are considered to be derived from the friction force, $f_{\mathrm{H}}$ and the horizontal bearing force, $F_{\mathrm{H}}$, respectively.

\section{Summary}

In the present study, the capability of the neutron diffraction technique for the structural engineering studies on the reinforced concrete structure was discussed based on the bond stress evaluation between rebar and concrete. Several peaks appear in the bond stress distribution measured by neutron diffraction, showing the inhomogeneous bond variation along the embedded rebar. The horizontal bearing force on the rib face might increase the bond resistance around the transverse ribs, increasing

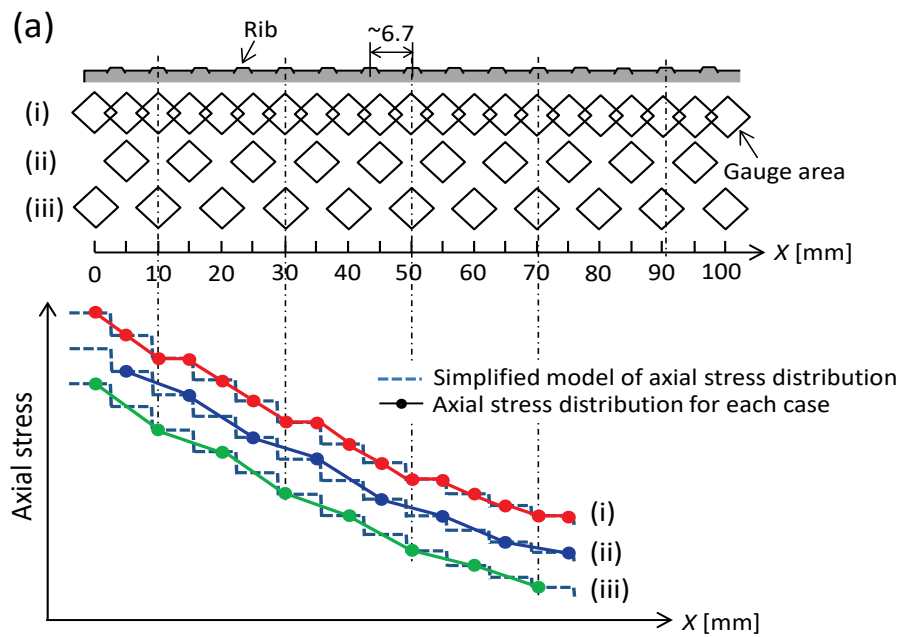

(b)

$f_{H}$ : Friction force at straight part $\mathrm{F}_{\mathrm{H}}$ : Horizontal bearing force at rib face $\mathrm{F}_{\mathrm{V}}$ : Vertical bearing force at rib face $\mathrm{F}_{\mathrm{N}}$ : Normal force on rib face from concrete

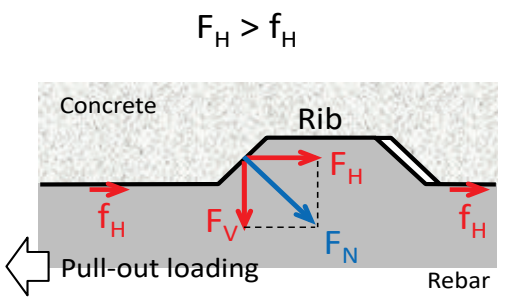

Fig. 5 (a) Schematic relationship between transverse rib and measurement positions and schamtic diagram of the axial stress distribution estimated for each spatial resolution. (b) Schamtic illustration of force working around a transverse rib.

the bond stresses locally. On the other hand, lower bond stresses are considered to be derived from weaker friction force acting at the straight part of rebar between transverse ribs. This result suggests that neutron diffraction technique with high spatial resolution can investigate local bond resistance caused by the transverse ribs. The bond stress distribution measured by the neutron diffraction technique is expected to bring detailed understanding of the bond mechanism between rebar and concrete for the reinforced concrete structures. 
The absorption coefficient of concrete is approximately $0.5 \mathrm{~cm}^{-1}$ which is about a half of iron one [5]. For the future, to use deuterated concrete would decrease the transmission and allow higher spatial resolution and allow us to see more detailed cyclic variation associated with the ribs.

The neutron diffraction experiments were performed using the TAKUMI engineering diffractometer at the J-PARC/MLF with the approval of the J-PARC Center as Proposal No. $2012 \mathrm{~B} 0058$.

\section{References}

[1] Y. Goto, Cracks formed in concrete around deformed tension bars, ACI Journal, 68 (1971) 244251.

[2] A. H. Nilson, Internal measurement of bond slip, J. Am. Concr. Inst., 69 (1979) 439-441.

[3] R. Tepfers, Cracking of concrete cover along anchored deformed reinforcing bars Magazine of Concrete Research 31 (1979) 3-12.

http://dx.doi.org/10.1680/macr.1979.31.106.3

[4] M. N. S. Hadi, Bond of high strength concrete with high strength reinforcing steel, The Open Civil Engineering Journal, 2 (2008) 143-147. http://dx.doi.org/10.2174/1874149500802010143

[5] H. Suzuki, M. Kanematsu, K. Kusunoki, Neutron diffraction studies on strain evaluation of rebar in reinforced concrete, Powder Diffraction, 24, Issue S1 (2009) S68-S71.

[6] K. Kusunoki, K. Kabayama, T. Mukai, Y. Hatanaka, H. Suzuki, A. Tasai, Experimental study on bonding action with neutron diffraction technique, 8CUEE CONFERENCE PROCEEDINGS, (2011) 701-705.

[7] H. Suzuki, K. Kusunoki, Y. Hatanaka, T. Mukai, A. Tasai, M. Kanematsu, K. Kabayama, S. Harjo, Measuring strain and stress distributions along rebar embedded in concrete using time-offlight neutron diffraction, Measurement Science and Technology, 25 (2014) 025602. http://dx.doi.org/10.1088/0957-0233/25/2/025602

[8] H. Suzuki, K. Kusunoki, M. Kanematsu, A. Tasai, Y. Hatanaka, N. Tsuchiya, S.C. Bae, S. Shiroishi, S. Sakurai, T. Kawasaki, S. Harjo, Application of neutron stress measurement to reinforced concrete structure, JPS Conference Proceedings, 8 (2015) 031006.

http://dx.doi.org/10.7566/jpscp.8.031006

[9] F. I. Faiyadh, Bond characteristics of oil saturated concrete, Int. J. Cement Composites and Lightweight Concrete, 7 (1985) 115-131. http://dx.doi.org/10.1016/0262-5075(85)90067-3

[10] S. W. Lee, K. H. Tan, E. H. Yang, Bond-slip response of reinforcing bars embedded in high performance fiber reinforced cement composites, Int. J. Civil Environmental, Structural, Construction and Architectural Engineering, 9 (2015) 626-631.

[11] S. Harjo, T. Ito, K. Aizawa, H. Arima, J. Abe, A. Moriai, T. Iwahashi, T. Kamiyama, Current status of engineering materials diffractometer at J-PARC, Materials Science Forum, 681 (2011) 443448.

http://dx.doi.org/10.4028/www.scientific.net/MSF.681.443

[12] R. Oishi, M. Yonemura, Y. Nishimaki, S. Torii, A. Hoshikawa, T. Ishigaki, T. Morishima, K. Mori, T. Kamiyama, Rietveld analysis software for J-PARC, Nuclear Instruments and Methods, A 600 (2009) 94-96. 